\title{
Gehalt des braunen Fettgewebes der Ratte an Noradrenalin und zyklischem AMP im Magnesiummangel
}

\author{
Von Th. Günther, J. Schmalbeck und H. J. Merker \\ Zentralinstitut für Biockemie und Biophysik, Physiologisch-chemisches Institut und II. Anatomisches Institut \\ der Freien Universität Berlin
}

(Eingegangen am 22. März 1973)

Herrn Prof. Dr. Dr. Ernst Schütte qum 65. Geburtstag gewidmet

\begin{abstract}
Nach 4 Wochen langer Mg-armer Ernährung war im braunen Fettgewebe, Herz und Leber der Ratte der Gehalt an Ado-3':5'-P um $20-40 \%$ angestiegen.

Gleichzeitig waren die Zahl und Größe der Noradrenalin-haltigen Vesikel und Granula im braunen Fettgewebe vermindert. Damit übereinstimmend war das fluoreszenzoptisch darstellbare Noradrenalin in den Vesikeln und Granula der sympathischen Nervenfasern verschwunden.

Für die Lipolyse und Mitochondrienschwellung im braunen Fettgewebe im Mg-Mangel wird ein Noradrenalin-Ado-3':5'-P-abhängiger Mechanismus diskutiert.
\end{abstract}

\section{The concentration of noradrenaline and grclic AMP in the brown fat tissue of the rat during magnesium deficiency}

After four weeks on a $\mathrm{Mg}$-deficient diet, the concentration of adenosine- $3^{\prime}, 5^{\prime}$ phosphate in the brown fat tissue, heart and liver of the rat showed an increase of $20-40 \%$. At the same time, the noradrenaline-containing vesicles and granules in the brown fat tissue were decreased in size and number; and the vesicles and granules of the sympathetic nerve fibres no longer contained noradrenaline as shown by the fluorescence-optical method.

It is proposed that noradrenaline and adenosine $3^{\prime}, 5^{\prime}$-phosphate are concerned in lipolysis and mitochondrial swelling in brown fat tissue in Mg-deficiency.

Nach chronisch $\mathrm{Mg}$-armer Ernährung kommt es, wenn die $\mathrm{Mg}$-Konzentration im Serum abgesunken ist, in den braunen Fettzellen zur Verringerung der Zahl und Größe der Fetteinschlüsse und zur Schwellung der Mitochondrien (1). Analoge Veränderungen findet man bei akuter Kältebelastung (1).

Die Wirkung des Kältestreß erfolgt über Freisetzung von Noradrenalin in den sympathischen Nervenfasern und Nervenfaserendigungen des braunen Fettgewebes, die dort reichlich vorhanden sind. Das hier freigesetzte Noradrenalin aktiviert die Adenylcyclase. Die resultierende höhere Konzentration von Ado- $3^{\prime}: 5^{\prime}-\mathrm{P}$ in den braunen Fettzellen erhöht die Lipaseaktivität direkt und vielleicht auch indirekt über eine Erhöhung der Fructosediphosphat-Konzentration. Die durch die Lipase abgespaltenen freien Fettsäuren bewirken - in noch nicht entschiedener Weise - die Entkopplung der oxydativen Phosphorylierung (2).

Für die analogen Veränderungen der braunen Fettzellen im $\mathrm{Mg}$-Mangel bietet sich eine entsprechende Erklärung an.

Zum Beweis haben wir im braunen Fettgewebe bei Mg-Mangel den Noradrenalin-Gehalt histochemisch und den Ado- $3^{\prime}: 5^{\prime}-$ P-Gehalt im Protein-Bindungstest bestimmt. Um zu prüfen, ob die Beeinflussung des Ado-3':5'-P-Gehaltes spezifisch für braunes Fettgewebe ist, haben wir Ado-3':5'-P zusätzlich in Leber, Herz und Muskel gemessen.

\section{Methodik}

$100 \mathrm{~g}$ schwere männliche Wistarratten wurden wie beschrieben (3) mit einer $\mathrm{Mg}$-armen bzw. $\mathrm{Mg}$-reichen Diät gefüttert.

In Äthernarkose wurde den Tieren das interscapuläre braune Fettgewebe, korrespondierende Proben aus Leber und Muskel (m. quadriceps) sowie das Herz entnommen und sofort in flüssigem $\mathrm{N}_{2}$ eingefroren.

Die Organe wurden nach Gisman (4) extrahiert und Ado-3': $5^{\prime}-P$ im Extrakt nach den Vorschriften der Testkombination (Fa. Boehringer, Mannheim, Bestellnummer 15289) bestimmt.

Das Noradrenalin in den sympathischen Nervenfasern wurde mit der Fluoreszenzmethode nach FALCK und Osman (5) dargestellt. Die elektronenmikroskopischen Bilder wurden wie bereits beschrieben (1) hergestellt.

Zum Vergleich mit früheren Untersuchungen wurden die Lipide des braunen Fettgewebes mit Sudan angefärbt. Im gleichen Schnitt haben wir die Cytochromoxydase nach Burstone (6) dargestellt.

\section{Ergebnisse}

In Ergänzung unserer früheren Untersuchungen über die Veränderungen des braunen Fettgewebes im $\mathrm{Mg}$ Mangel zeigt Tabelle 1 , daß der Gehalt an Ado-3': $5^{\prime}-\mathrm{P}$ der untersuchten Organe mit Ausnahme des Muskels um $20-40 \%$ angestiegen ist.

Der physiologische Aktivator der Adenylcyclase im braunen Fettgewebe ist Noradrenalin, das in Vesikeln und Granula der 'sympathischen Nervenfasern gespeichert und mittels Exocytose abgegeben werden kann. 
Tab. 1

Gehalt an Ado-3':5'-P in verschiedenen Organen $M g$-reich und $M g$-arm ernährter Ratten [ $\mu \mathrm{mol} / \mathrm{kg}$ Feuchtgewicht] Mittelwert \pm mittlerer Fehler des Mittelwertes, $\mathrm{n}=$ Zahl der Versuchstiere

\begin{tabular}{|c|c|c|c|c|}
\hline & $\begin{array}{l}\text { Braunes } \\
\text { Fettgewebe }\end{array}$ & Leber & Herz & Muskel \\
\hline Mg-reiche Diät & $\begin{array}{c}1,15 \pm 0,03 \\
(n=9)\end{array}$ & $\begin{array}{c}0,80 \pm 0,02 \\
(n=5)\end{array}$ & $\begin{array}{c}0,50 \pm 0,02 \\
(n=6)\end{array}$ & $\begin{array}{c}0,40 \pm 0,04 \\
(n=5)\end{array}$ \\
\hline Mg-arme Diät & $\begin{array}{c}1,48 \pm 0,05 \\
(n=9)\end{array}$ & $\begin{array}{c}0,98 \pm 0,02 \\
(n=5)\end{array}$ & $\begin{array}{c}0,67 \pm 0,04 \\
(n=6)\end{array}$ & $\begin{array}{c}0,43 \pm 0,02 \\
(n=8)\end{array}$ \\
\hline
\end{tabular}

Diese Vesikel und „dense core granula“ (kleinere Vesikel, die Granula enthalten) finden sich in den nackten, d. h. Hüllzell-freien Endabschnitten und in umschriebenen Bezirken im Verlauf der Axone. Die Axone verdicken sich an diesen Stellen, entfalten dadurch die Mesaxone und grenzen frei an den Interzellularraum. Unter den Bedingungen des Mg-Mangels sind diese Stellen geringer ausgebuchtet und enthalten weniger Vesikel. Die Axone liegen jetzt tiefer in den Schw'ANNschen Zellen. In den Endabschnitten ist im $\mathrm{Mg}$-Mangel die Zahl der Vesikel und der „dense core granula" ebenfalls verringert. Der Gehalt an elektronen-

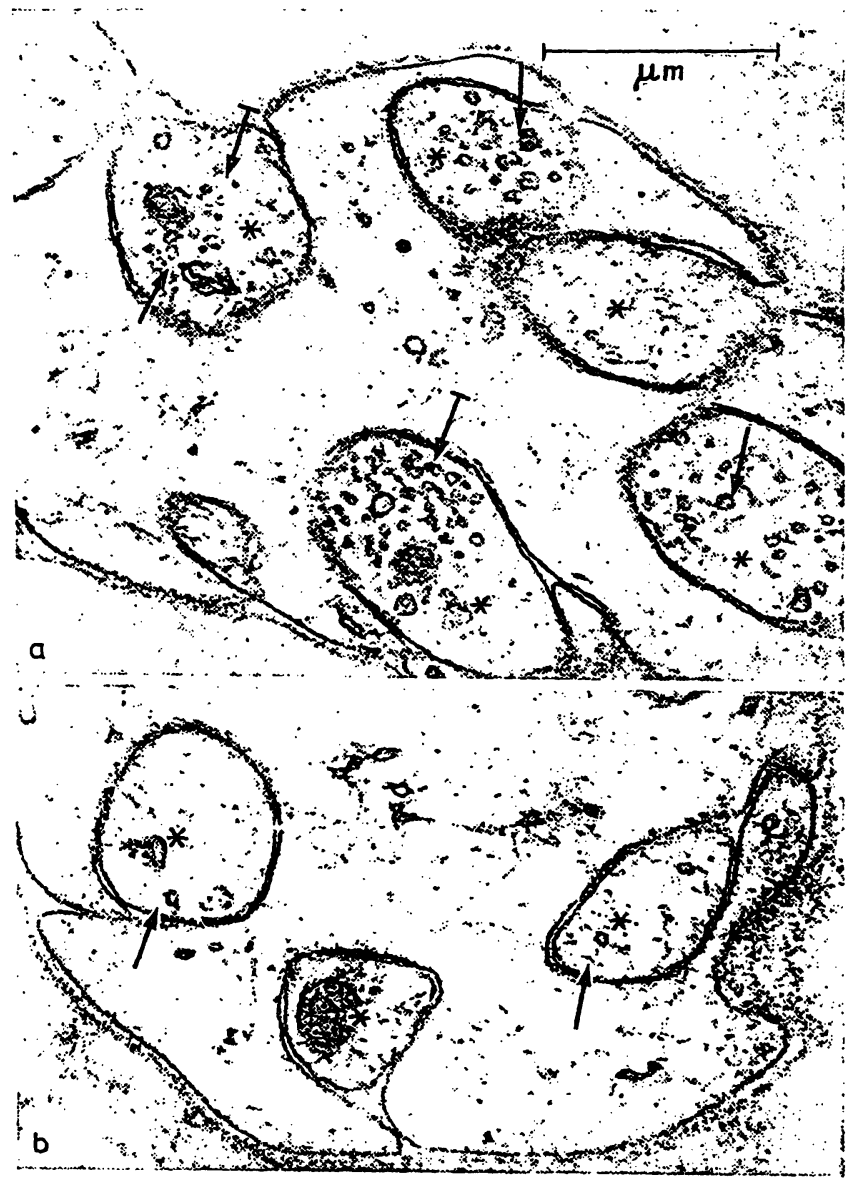

Abb. 1

Elektronenmikroskopisches Bild von einem Querschnitt durch eine sympathische Nervenfaser des braunen Fettgewebes der Ratte mit mehreren Axonen (*)
Vergrößerung: $1: 24000$

a) Normaltier: Axone mit zahlreichen Vesikeln $(\downarrow)$ und "dense core b) 4 Wochen Mg-arm ernährte Ratte: Mg-arm ernährte Ratte: Die Axone enthalten nur noch
wenige Vesikel und "dense core granula“" dichtem Material in diesen Vesikeln ist $z$. T. vermindert (Abb. 1).

Das gleiche Ergebnis zeigt sich mit der histochemischen Fluoreszenzmethode (Abb. 2). Durch diese Methode lassen sich die vesikelreichen, d. h. die Noradrenalinhaltigen Abschnitte der sympathischen Nervenfasern als fluoreszierende Plaques, die häufig zu Ketten geordnet sind, darstellen. Bei kurzfristigem Mg-Mangel ist die Fluoreszenz des in Vesikeln gespeicherten Noradrenalins vermindert, nach vierwöchiger $\mathrm{Mg}$ Mangel-Ernährung fehlt sie vollständig.

Das Ausmaß der Veränderungen wird durch die gleichzeitige Anfärbung der Lipideinschlüsse belegt (Abb. 3). Nach vierwöchiger $\mathrm{Mg}$-armer Ernährung sind keine Fetttropfen in den braunen Fettzellen mehr zu sehen. Die in den gleichen Abbildungen sichtbare Darstellung der Cytochromoxydase, die das geschwindigkeits-

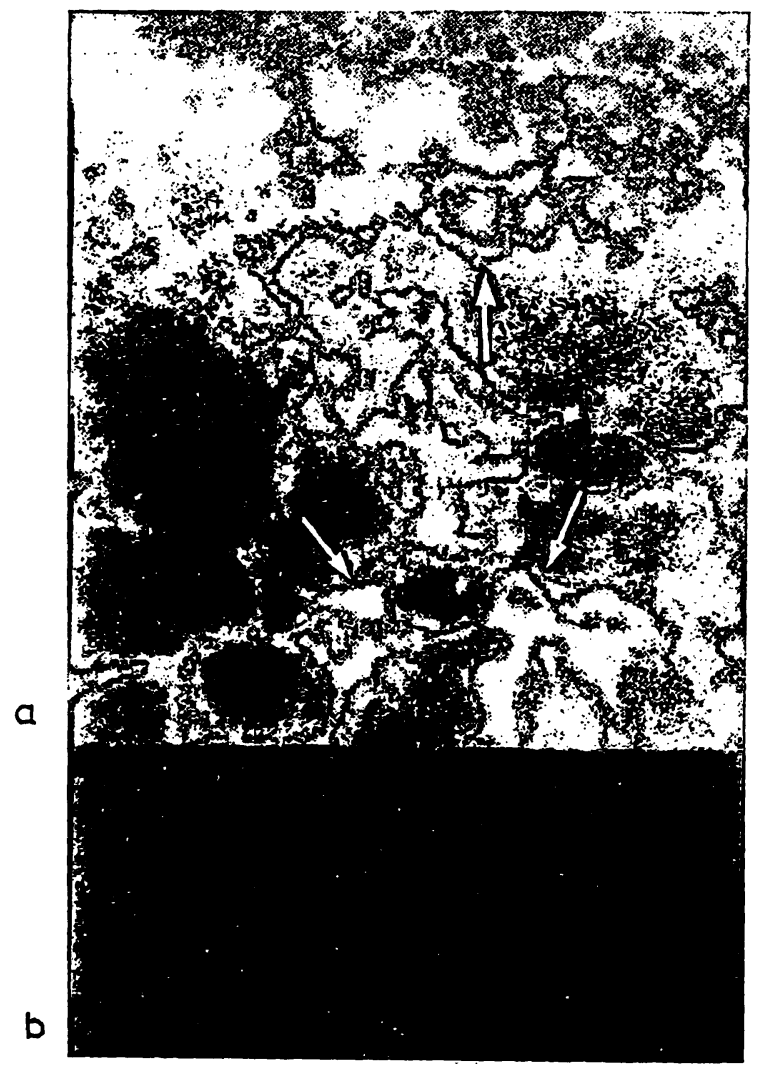

Abb. 2

Fluoreszenzoptische Darstellung der Noradrenalin-haltigen Abschnitte in sympathischen Nervenfasern des braunen Fettgewebes a) Normaltier: Zahlreiche fluoreszierende Plaques ( $\downarrow$ ) b) 4 Wochen Mg-arm ernährte Ratte: keine Fluoreszenz Vergrößerung: $1: 350$ 

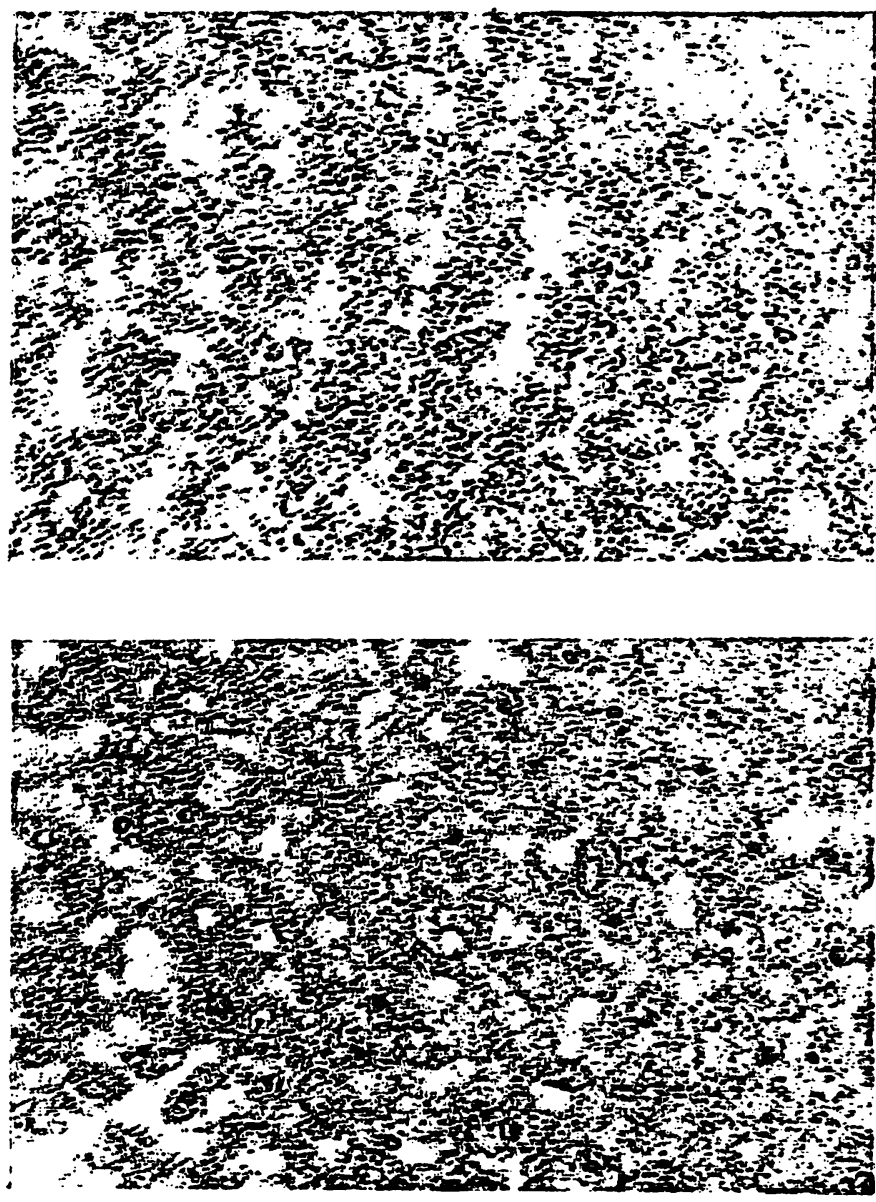

Abb. 3

Histochemische Darstellung der Cytochromoxydase und Färbung der ben: Normaltier: Fetteinschlüsse (Sudan)

ahlreiche große (rote) Fetteinschlüsse und zahlreiche (dunkle) Mitochondrien

unten: 4 Wochen $\mathrm{Mg}$-arm ernährte Ratte: keine Sudan-positiven Einschlüsse. Cytochromoxydase-positive Mitochondrien wie oben Vergrößerung: 1:560

bestimmende Enzym des mitochondrialen Elektronentransportsystems ist, läßt keine signifikanten Unterschiede erkennen. Im $\mathrm{Mg}$-Mangel sind infolge der Schwellung größere Mitochondrien zu sehen.

\section{Diskussion}

Die Bestimmung des Ado-3':5'-P-Gehaltes ergab, daß die Veränderungen der braunen Fettzellen im $\mathrm{Mg}$ -

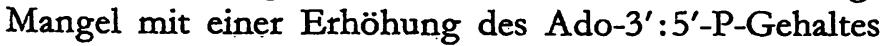
einhergehen und deshalb wie bei der physiologischen Funktion der braunen Fettzellen von Ado-3': $5^{\prime}-\mathrm{P}$ verursacht sein können.

Die Zunahme des Ado-3':5'-P bei Mg-Mangel im braunen Fettgewebe beträgt nur etwa $30 \%$, so daß man diskutieren kann, ob diese Zunahme bei Zugrundelegung eines reversiblen Enzym-Effektor-Mechanismus genügt, die Lipase ausreichend $\mathrm{zu}$ aktivieren und somit die sichtbaren Effekte (1) auszulösen. Es ist jedoch zu bedenken, daß braune Fettzellen mit ihren zahlreichen Organellen und Fetteinschlüssen ein gekammertes System darstellen und die Konzentration des Ado-3': $5^{\prime}-\mathrm{P}$ an seinem Wirkungsort sich deshalb stärker verändern kann, als aus der durchschnittlichen Konzentration im Gewebe folgen würde. Darüberhinaus besteht die Möglichkeit, daß Ado-3':5'-P z. B. zusammen mit Calcium, dessen intrazellulärer Gehalt im $\mathrm{Mg}$-Mangel ansteigt (7), eine stärkere Wirkung entfaltet (8).

Im Mg-Mangel war bei histochemischer Darstellung der Noradrenalin-Gehalt vermindert. Mit dieser histochemischen Fluoreszenzmethode wird nur das in Vesikeln konzentrierte Noradrenalin nachgewiesen (5). Sie ist nicht spezifisch. So bilden besonders primäre aromatische Amine, wie Dopamin oder 5-Hydroxytryptamin, ein fluoreszierendes DihydroisochinolinDerivat. Adrenalin reagiert langsamer (5). Die Methode weist im braunen Fettgewebe Noradrenalin nach, da andere primäre Amine nicht im gleichen $\mathrm{MaBe}$ (bis zu $10000 \mathrm{fach})$ in den Vesikeln angereichert werden, und diese Substanzen eine andere Farbe des Fluoreszenzlichtes aufweisen würden (5).

Im Mg-Mangel waren elektronenmikroskopisch mit dem Ergebnis der Fluoreszenzmethode übereinstimmend die Zahl der Vesikel und "dense core granula" und in einigen Vesikeln wahrscheinlich auch der Gehalt an Noradrenalin vermindert. Hieraus kann man auf eine vermehrte Ausschleusung (Exocytose) der Vesikel und/oder eine verminderte Wiederaufnahme des Noradrenalins in die Vesikel schließen. Für die erste Möglichkeit spricht, daß bei erniedrigter extrazellulärer $\mathrm{Mg}$-Konzentration vermehrt Vesikulationsprozesse sichtbar sind (9). Für die Hemmung der Wiederaufnahme von Noradrenalin in die Vesikel spricht, daß für die Aufnahme Mg und ATP benötigt wird (10). Es ist also wahrscheinlich, daB diese beiden $\mathrm{Mg}$-abhängigen Prozesse zu einer höheren Konzentration an freiem Noradrenalin im braunen Fettgewebe führen, was aber mit der Fluoreszenzmethode nicht mehr darstellbar ist, da mit freigesetztem und im braunen Fettgewebe verteiltem Noradrenalin die untere Nachweisgrenze der Methode unterschritten wird (5).

$\mathrm{Ob}$ also eine vermehrte Bindung von Noradrenalin an seinen spezifischen Rezeptor auf der Zellmembran stattfindet, ist möglich, aber nicht bewiesen. Die früher diskutierte, zusätzliche (direkte oder indirekte) Wirkung einer herabgesetzten Serum-Mg-Konzentration auf die Aktivität der Adenylcyclase (1) oder eine mit dem erniedrigten extrazellulären $\mathrm{Mg}$ einhergehende

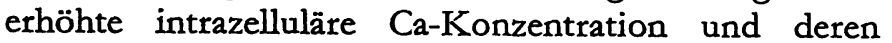
vielfältige Wirkungen auch in Verbindung mit Ado$3^{\prime}: 5^{\prime}-\mathrm{P}(10)$ sind daher nicht auszuschließen.

Die Erhöhung der Ado-3':5'-P-Konzentration im $\mathrm{Mg}$-Mangel ist nicht auf das braune Fettgewebe begrenzt, sondern fand sich auch bei anderen Geweben. Da Ado-3':5'-P z. B. die Kontraktion von glatten Muskelfasern fördert (10), könnten die mannigfaltigen klinischen Erscheinungen des $\mathrm{Mg}$-Mangels (im vasculären, cardialen und visceralen Bereich (11)) biochemisch hierauf zurückgeführt werden. 


\section{Lhernatue}

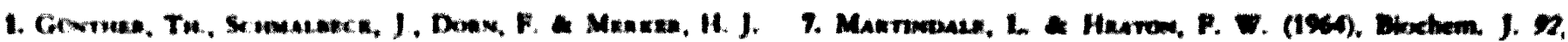

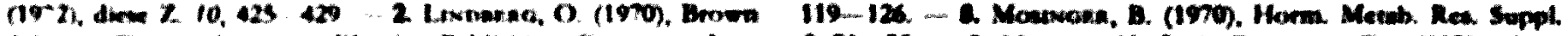

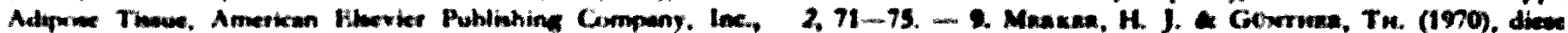

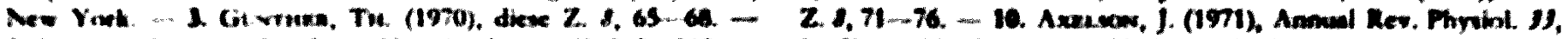

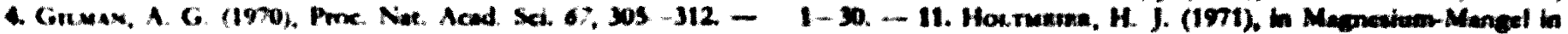

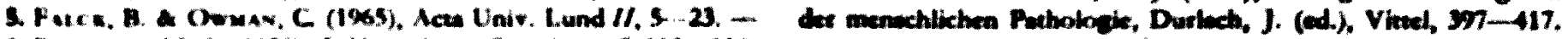

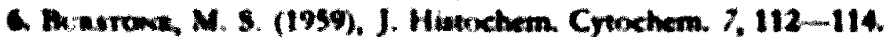

Prot. Dr. Th. Comber 1000 Bestin 33

Amimallea 22 